\title{
Quantitative Analyse der Krebsentstehung
}

\author{
Von Hermann Druckrey* und Karl Küpfmüller* \\ (Z. Naturforschg. 3 b, 254-266 [1948]; eingegangen am 5. Juni $1947 * *$ )
}

In quantitativen Versuchen an 700 Ratten mit dem krebserregenden Farbstoff „Buttergelb" ergaben sich folgende Beziehungen zwischen der Dosis und der Wirkung:

1. Die Latenzzeit bis zum Auftreten der ersten Lebergeschwülste ist eine einfache Funktion der täglich gegebenen Dosis.

2. Zur Geschwulsterzeugung ist eine bestimmte Gesamtdosis „Buttergelb“ erforderlich, ohne Rücksicht darauf, über welche Zeit sie zwischen 34 Tagen und einem Jahr verteilt wird. Die Latenzzeit ist in diesem Bereich der Höhe der täglichen Dosis umgekehrt proportional.

3. Bei längerer Versuchsdauer, die sich über die ganze Lebenszeit der Ratten erstreckt, genügt sogar eine kleinere Gesamtdosis. Das deutet auf eine Zunahme der Krebs-Disposition im Alter hin.

4. Die Größe der zur Krebsauslösung notwendigen Gesamtdosis „Buttergelb“ kann als $\mathrm{Maß}$ für die Größe der vorhandenen Krebsdisposition dienen.

5. Die Streuung der Versuchsergebnisse ist mit nicht mehr als $10 \%$ auffällig gering. Daraus ergibt sich die Folgerung, daß zum Geschwulstwachstum eine Mindestzahl von erzeugten Krebszellen vorhanden sein muß.

6. Die Effekte auch der kleinsten Einzeldosen bleiben bei diesem Farbstoff über die ganze Lebenszeit der Ratten voll summationsfähig bestehen.

7. Da in dieser Zeit häufig Zellteilungen stattgefunden haben, müssen die gesetzten Effekte auf die Tochterzellen übertragen worden sein. Die Zellbestandteile, an denen die cancerogene Wirkung ansetzt, haben also die Fähigkeit zur Selbstproduktion, d.h. der Effekt ist erblich.

8. Nach den vorliegenden Ergebnissen bestehen zwischen krebsiger Entartung und genetischer „Mutation“ zumindest quantitative Unterschiede.

9. Der einfache reaktionskinetische Ansatz erweist sich für die Wirkung cancerogener Agenzien auf den fraglichen Zellbestandteil anwendbar. Er erklärt eine Reihe bisher unerklärbarer Phänomene zwanglos und führt zu weiteren klaren Problemstellungen.

10. Die „Treffertheorie“" wird auf den gleichen reaktionskinetischen Ansatz zurückgeführt.

11. Die krebserzeugende Wirkung unterliegt genau so bekannten physikalisch-chemischen Gesetzmäßigkeiten wie jede andere Wirkung auch.

12. Der Farbstoff „Buttergelb“ ist bei dauernder Zufuhr auch in kleinsten Dosen schädlich und deshalb als Lebensmittelfarbstoff abzulehnen.

$\mathrm{K}$ in o sit $\mathrm{a}^{\mathbf{1}}$ hatte gefunden, daß das als Lebensmittelfarbstoff viel verwendete Dimethylaminoazobenzol (,Buttergelb“) an Ratten Leberkrebs erzeugt. Diese Beobachtung wurde bestätigt ${ }^{2}$, wobei sich ergab, daß die krebserzeugende Wirkung nicht, wie zunächst vermutet wurde, einer Verunreinigung des Farbstoffs zukam, sondern auch dem hochgereinigten Produkt. Daraufhin empfahlen wir ein Verbot der Verwendung des „Buttergelbs“ als Lebensmittelfarbstoff, das vorübergehend auch bestand.

Die praktische Bedeutung, die dieser verhäng-

* (17b) Freiburg (Breisgau), Chirurg. Klinik d. Universität (Direktor: Prof. E. Rehn), und Stuttgart, Hackländerstr. 19.

** Die Arbeit kann aus zeitbedingten Umständen erst jetzt erscheinen. nisvolle Farbstoff damit hatte, veranlaßte uns dann zur Prüfung der Frage, ob kleine Dosen des krebserregenden Stoffs auch bei dauernder Zufuhr als unschädlich angesehen werden können. Gleichzeitig interessierte uns das theoretische Problem, ob bei diesem krebserregenden Stoff quantitative Beziehungen zwischen Dosis und Wirkung nachweisbar sind, seine Wirkung sich also grundsätzlich in allgemeine pharmakologische Gesetzmäßigkeiten fügt oder nicht. Über erste Ergebnisse hat der eine ${ }^{3}$ von uns kurz berichtet. Dabei wurde gezeigt, aus welchem Grunde die bisher mit cancero-

1 R. K i n o s it a, Transact. Soc. path. jap. 27, 665 [1937].

${ }^{2}$ N. B r o c k, H. D r u ck rey u. H. Hamper l, Z. Krebsforschg. 50, 431 [1940].

${ }^{3}$ H. D r u c k rey, Klin. Wschr. 22, 532 [1943]. 
genen Kohlenwasserstoffen unternommenen quantitativen Untersuchungen ${ }^{4}$ kein klares Resultat liefern konnten.

Entsprechende Versuche mit verschiedenen der cancerogenen aromatischen Kohlenwasserstoffe, mit Benzpyren, Methylcholanthren und Dibenzanthracen, sind mehrfach durchgeführt worden ${ }^{4,5}$. Die erwartete quantitative Beziehung zwischen Dosis und Wirkung hat sich dabei nur andeutungsweise hinsichtlich der Häufigkeit der Geschwulstbildungen ${ }^{5}$ ergeben, nicht aber für die Latenzzeit, die bei manchen Versuchen sogar für mehrere Dosierungen praktisch die gleiche war. Dieser scheinbare Widerspruch zu allen bisherigen Erfahrungen der Pharmakologie ist durch eine ungenügende Beachtung der pharmakologischen Gesetzmäßigkeiten bedingt und klärt sich auf einfache Weise ${ }^{6}$.

Eine pharmakologische Wirkung ist nicht von der Höhe der Dosis unmittelbar abhängig, sondern von der Konzentration des Pharmakons am Wirkungsort. Nur insoweit diese Konzentration von der Dosis bestimmt wird, hat die Dosis Einfluß auf die Wirkung. Die genannten aromatischen Kohlenwasserstoffe sind nun in Körperflüssigkeiten fast unlöslich. Ihre Löslichkeit kann nach eigenen Erfahrungen mit etwa $10^{-7}$ angenommen werden. Eine Dosis von $1 \mathrm{mg}$ erzeugt also noch in $10000 \mathrm{~cm}^{3}$ eine volle Sättigung. Bedenkt man ferner, daß eine Maus im Durchschnitt 20 g oder eine Ratte $200 \mathrm{~g}$ wiegt, so heißt das, daß in einem Bereich von 0,002 bzw. 0,02 mg pro Dosis an aufwärts bis zu den höchsten angewendeten Dosen von mehr als $20 \mathrm{mg}$, also über 3-4 Größenordnungen, gar keine quantitative Abhängigkeit der Wirkung von der Dosis erwartet werden kann. Trotz der verschiedenen Dosis ist nämlich die Konzentration des Pharmakons am Wirkungsort hier stets die gleiche, nämlich die einer gesättigten Lösung. Erst unterhalb dieses Bereichs können quantitative Beziehungen zwischen Dosis und Wirkung erwartet werden. Ihre Exaktheit hängt naturgemäß von der .Genauigkeit der Dosierung ab, deshalb sind Pinselungsversuche nicht sehr geeignet. Darüber hinaus schwankt das Lösungsvermögen der Tiere sehr und bedingt starke Streuungen. Dennoch zeigen die Angaben der Literatur, daß auch bei den cancerogenen aromatischen Kohlenwasserstoffen ganz klare Beziehungen zwischen der Dosis und Wirkung auftreten, wenn diese Besonderheiten der Löslichkeit berücksichtigt werden. So fanden z. B. B r y a n und $\mathrm{Sh}$ i $\mathrm{m} \mathrm{k} \mathrm{in}^{5}$ an $\mathrm{C}_{3} \mathrm{H}$-Mäusen eine feste Abhängigkeit der Geschwulsthäufigkeit von 0 bis $100 \%$ in einem Dosierungsbereich zwischen $0,00098 \mathrm{mg}$ und $0,062 \mathrm{mg} \mathrm{Di}$ benzanthracen, dagegen zwischen $0,062 \mathrm{mg}$ und $1 \mathrm{mg}$ keine, die Häufigkeit bleibt $100 \%$. FriedrichFreks a ${ }^{5}$ beobachtete bei Pinselungsversuchen mit Methylcholanthren und Benzpyren an Mäusen eine Dosisabhängigkeit der Geschwulsthäufigkeit und der Latenzzeit im Bereich von $0,5-1 \mathrm{mg}$.

4 W. F. Dunning, M. R. Curtis u. F. C. W o o d, Amer. J. Cancer 39, 70 [1940]; G. R o u s s y, P. u. N. Gu ér i n, Bull. Assoc. franç. Etude Cancer 30, 66 [1942].

\section{Versuche}

Die Versuche wurden an dem unter den gegebenen Verhältnissen größtmöglichen Tiermaterial von über 700 Ratten vorgenommen. Die Tiere stammten teils aus einem erbähnlichen Stamm von K r ö n in g ( $f a h l$ sepia-wei $\beta$ ), teils aus der heterozygoten Zucht eines Lieferanten. Ihr Alter betrug bei Versuchsbeginn etwa 100 Tage. Als krebserregenden Stoff verwendeten wir Dimethylamino-azobenzol pro analysi von Merck und von Schering. Die Zufuhr erfolgte per os. Zwecks genauer Dosierung wurde der Farbstoff sorgfältig mit Weizenmehl verrieben; aus diesem Mehl wurde dann, unter Zusatz der als notwendig berechneten Mengen an Vitaminen, Eiweiß, Fett und Mineralstoffen, Brot mit verschieden dosiertem „Buttergelb“Gehalt gebacken. Davon erhielten die Tiere täglich abgewogene Mengen. Etwa nicht gefressene Reste wurden zurückgewogen und bei der Dosierung des nächsten Tages berücksichtigt. Da die Ratten auch bei Anwendung von Köderstoffen nicht mehr als 5 mg „Buttergelb" am Tage in der Nahrung aufnahmen, gaben wir höhere Dosen, in Stärkekleister suspendiert, mit der Injektionsspritze durch eine Schlundsonde. Diese Sorgfalt wendeten wir an, um eine Streuung der Versuchsergebnisse durch Dosierungsfehler und Ernährungsunterschiede möglichst klein zu halten, so daß eine im Versuch gefundene Streuung allein auf die individuelle Variation des Tiermaterials bezogen werden konnte.

Die Aufteilung der Ratten für diese quantitativen Versuche erfolgte in sechs Versuchsgruppen mit geometrisch ansteigender Dosierung und in einer Kontrollgruppe. Die erste Versuchsgruppe erhielt 0,1 , die zweite 0,3 , die dritte 1 und die vierte $3 \mathrm{mg}$ „Buttergelb" pro Tag und $200 \mathrm{~g}$ Tier, in Brot eingebacken, die fünfte und sechste erhielt $10 \mathrm{bzw} .30 \mathrm{mg} / \mathrm{Tag}$ durch die Schlundsonde. Später kam noch eine kleine Versuchsgruppe mit $20 \mathrm{mg} / \mathrm{Tag}$ hinzu, um das Ergebnis der benachbarten Gruppen zu prüfen. Die Kontrollen und die sondierten Tiere erhielten die gleiche Standardfütterung wie die andern Versuchsgruppen. Höhere Dosen als $30 \mathrm{mg} / \mathrm{Tag}$ konnten nicht gegeben werden, weil sie zu toxisch waren. Schon bei dieser Dosierung betrug die mittlere Lebenserwartung infolge der Giftwirkung nur noch 32 Tage. Die in einem Tage wirkende „mittlere tödliche Dosis“ wurde zu $58 \mathrm{mg}$ „Buttergelb" bestimmt. In den Versuchsgruppen mit den hohen Dosierungen starb daher ein großer Teil der Tiere, bevor die ersten Geschwülste auftraten.

$\mathrm{Zu}$ verschiedenen Zeiten wurden einige Tiere in jeder Versuchsgruppe getötet und ihre Leber histologisch untersucht, um den Verlauf der Wirkung verfolgen $\mathrm{zu}$ können. Bei allen andern Tieren warteten wir den Eintritt des natürlichen Todes ab, den wir durch sorgfältige Pflege der Tiere hinauszuzögern

5 G. Miescher, F. Almasy u. F. Z e hend e r, Schweiz. med. Wschr. 71, 1002 [1941]; W. R. B r y a n u. M. B. S h i m k i n, J. Nat. Canc. Inst. 4, 25 [1943]. - H. Friedri c h- Freks a, Biol. Zbl. 60, 498 [1940].

${ }_{6}$ H. D r u c k r e y, Klin. Wschr. 22, 532 [1943]. 
versuchten. Nach dem Tode erfolgte eine vollständige Sektion und eine genaue histologische Untersuchung der Leber. Diese Untersuchungen wurden von Hrn. Prof. H. H a m p e r l (Salzburg) durchgeführt. Über sie wird gesondert berichtet werden.

\section{Ergebnisse}

Die kleinen Dosen von 0,1 und 0,3 mg „Buttergelb" erzeugten keine Geschwülste, obwohl sich die Versuchsdauer bis zu 877 Tagen erstreckte. Uns interessierte jedoch nicht nur ein etwaiger positiver Befund, sondern ebenso der viel schwerer feststellbare negative, also die Frage: Welche Dosen erzeugen auch bei fortgesetzter Gabe über die ganze Lebenszeit keinen Krebs? Aus diesem Grunde waren in diesen beiden Versuchsgruppen allein über 300 Ratten angesetzt worden. Der negative Ausgang hinsichtlich der Geschwulsterzeugung erscheint also genügend gesichert. Dagegen traten bei Tieren beider Gruppen nach längerer Behandlungsdauer (Tab. 1) Veränderungen im histologischen Bild der Leber auf, wie Kernunruhe und Gallengangswucherungen, die nach unseren früheren ${ }^{2}$ und jetzigen Erfahrungen mit zwar beträchtlicher zeitlicher Streuung, aber doch in einem gewissen Abstand der Geschwulstbildung vorausgehen. Darauf kommen wir später zurück.

In den anderen Versuchsgruppen mit höheren Einzeldosen entstanden insgesamt 35 genau untersuchte Geschwülste, teils Adenome, teils Carcinome der Leber. Davon entfielen je 3 auf die mit 10, 20 und $30 \mathrm{mg} /$ Tag behandelten Gruppen, 20 auf die mit $3 \mathrm{mg}$ und 6 auf die mit $1 \mathrm{mg}$ behandelte Gruppe. Geschwülste in anderen Organen als der Leber haben wir bei einem Beobachtungsgut von mehr als 1000 Ratten nicht gesehen. Die Ergebnisse sind in Tab. 1 wiedergegeben.

Bei der Angabe der Latenzzeit ist das verschiedene Alter der Geschwülste durch eine Korrektur berücksichtigt worden, weil es in den Versuchen mit kurzer Latenzzeit einen erheblichen Fehler bedingen würde. Die Größe dieser Korrektur beträgt hier stets 7 Tage, in den anderen Versuchen, je nach Gewicht des Tumors, 5 bis 25 Tage.

Die in Tab. 1 wiedergegebenen Ergebnisse zeigen eine klare Beziehung zwischen der Höhe der täglichen Dosis $C$ und der Latenzzeit $t$ bis zum Auftreten der Geschwülste. Diese entstanden um so früher, je höher die tägliche Dosis war, bis zu der außerordentlich kurzen Latenzzeit von nur $34 \mathrm{Ta}$ gen. Bei den kleinen Dosen traten sie dagegen erst nach etwa 2 Jahren auf, fielen also kaum noch in

\begin{tabular}{|c|c|c|c|c|c|c|}
\hline \multirow{3}{*}{$\begin{array}{c}C \\
\text { Dosis } \\
\mathrm{mg} / \mathrm{Tag}\end{array}$} & \multirow{3}{*}{$\begin{array}{c}n \\
\\
\text { Tiere } \\
\text { Zahl }\end{array}$} & \multicolumn{3}{|c|}{$\begin{array}{c}t \\
\text { Latenzzeit }\end{array}$} & \multirow{3}{*}{$\begin{array}{c}C t \\
\underset{\substack{\text { Gesamt- } \\
\text { dosis }}}{\mathrm{mg}}\end{array}$} & \multirow{3}{*}{ 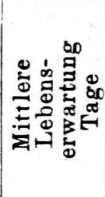 } \\
\hline & & \multirow{2}{*}{ 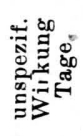 } & \multicolumn{2}{|c|}{$\begin{array}{l}\text { Geschwulst- } \\
\text { entstehung }\end{array}$} & & \\
\hline & & & $\begin{array}{c}\text { unkorr. } \\
\text { Tage }\end{array}$ & $\begin{array}{l}\text { korr. } \\
\text { Tage }\end{array}$ & & \\
\hline 0 & 120 & - & - & - & - & 500 \\
\hline 0,1 & 158 & 600 & - & - & - & 500 \\
\hline 0,3 & 148 & 420 & - & $=800$ & $>240$ & 500 \\
\hline 1 & 169 & 300 & 717 & 705 & 688 & 500 \\
\hline 3 & 70 & 140 & 3 tis & 350 & 1050 & 500 \\
\hline 10 & 30 & 30 & 102 & 95 & 950 & 86 \\
\hline 20 & 15 & 15 & 5 & 52 & 1040 & 52 \\
\hline 30 & 30 & 8 & 41 & 34 & 1020 & 32 \\
\hline
\end{tabular}

Tab.1. Krebserzeugung durch „Buttergelb“. Beziehung zwischen der Höhe der täglichen Dosis $C$ und der Dauer der Latenzzeit $t$ bis zum Auftreten der Geschwïlste.

die höchste Lebenserwartung der Ratten hinein. Die Gesamtdosis „Buttergelb“, die für die Auslösung der Geschwülste notwendig ist, ist durch das Produkt aus $C$ und $t$ gegeben. Sie ist, wenn zunächst vom 1-mg-Versuch abgesehen wird, unabhängig von der Höhe der Einzeldosis und der Behandlungsdauer und beträgt in allen Versuchen ziemlich genau 1000 mg „Buttergelb“. Dieses Ergebnis deckt sich völlig mit dem Ergebnis unserer früher veröffentlichten Versuche ${ }^{2}$ mit „Buttergelb“.

Die Berechnung der Versuchsergebnisse ist zwangsläufig dadurch gestört, daß in der langen Versuchszeit ein hoher Prozentsatz der Tiere interkurrent stirbt. Um diese Störung zu eliminieren, haben M i e scher und Mitarbb. ${ }^{5}$ vorgeschlagen, die Versuchsergebnisse auf die Tierzahl zu beziehen, die an dem Tag vorhanden war, an dem die ersten positiven Fälle auftreten (Versuchsbeginn) und diese Zahl gleich $100 \% \mathrm{zu}$ setzen. Dieser Vorschlag geht also von der Annahme aus, daß die vom Versuchsbeginn bis zum jeweiligen Stichtag gestorbenen negativen Tiere bei ihrem Weiterleben mit gleicher Wahrscheinlichkeit an Krebs erkrankt wären, wie die Tiere, die tatsächlich weiterleben. Der Gang der Rechnung ist folgender :

Ist $p$ die Zahl der an einem Stichtag anfallenden positiven Tiere (hier jeweils 1 ) un $1 n$ die Gesamtzahl der an diesem Tage noch lebenden (positiven und negativen) Tiere, so wäre bei Versuchsbeginn in einem Idealbestand von $N=100$ Tieren die (\%-) Zahl der positiven Fälle

$$
P=\frac{p}{n} N
$$

Bei den späteren Stichtagen sind nur die inzwischen angefallenen $P \%$ positiven Fälle vom Anfangswert der Tierzahl $(N=100)$ in Abzug zu bringen. Damit ist für jeden Stichtag

$$
N=100 \%-P \text {. }
$$




\begin{tabular}{|c|c|c|c|c|c|c|c|c|}
\hline \multirow{2}{*}{$\begin{array}{c}D \\
\text { Gesamtdosis } \\
\mathrm{mg}\end{array}$} & $\begin{array}{c}C \\
\text { Einzeldosis }\end{array}$ & $p$ & positive Fälle & $\Sigma$ & \multirow{2}{*}{$\begin{array}{c}N \\
\text { negative Fälle } \\
\%\end{array}$} & \multirow{2}{*}{$\begin{array}{c}n \\
\text { Gesamtzahl } \\
\text { Tiere }\end{array}$} & \multirow{2}{*}{$\begin{array}{c}\sigma \\
\text { Streuung } \\
\pm \mathrm{mg}\end{array}$} & \multirow{2}{*}{$\begin{array}{c}n B \\
\text { Gewicht der } \\
\text { Beobachtung }\end{array}$} \\
\hline & $\mathrm{mg}$ & Tiere & $\%$ & $\%$ & & & & \\
\hline $\begin{array}{r}1 \\
800\end{array}$ & - & $\begin{array}{l}0 \\
0\end{array}$ & $\begin{array}{l}0 \\
0\end{array}$ & $\begin{array}{l}0 \\
0\end{array}$ & $\begin{array}{l}0 \\
0\end{array}$ & $\begin{array}{l}75 \\
35\end{array}$ & & \\
\hline $\begin{array}{r}850 \\
940 \\
980 \\
990 \\
1020 \\
1020 \\
1020 \\
1050 \\
1050\end{array}$ & $\begin{array}{l}10 \\
10 \\
20 \\
30 \\
30 \\
20 \\
10 \\
20 \\
30\end{array}$ & $\begin{array}{l}1 \\
1 \\
1 \\
1 \\
1 \\
1 \\
1 \\
1 \\
1\end{array}$ & $\begin{array}{r}4,5 \\
5,3 \\
5,6 \\
6,5 \\
6,5 \\
7,2 \\
7,2 \\
14,3 \\
21,5\end{array}$ & $\begin{array}{r}4,5 \\
9,8 \\
15,4 \\
21,9 \\
28,4 \\
35,6 \\
42,8 \\
57,1 \\
78,6\end{array}$ & $\begin{array}{l}100 \\
95,5 \\
90,2 \\
84,6 \\
78,1 \\
71,6 \\
64,4 \\
57,2 \\
42,9 \\
21,4\end{array}$ & $\begin{array}{r}22 \\
18 \\
16 \\
13 \\
12 \\
10 \\
9 \\
4 \\
2\end{array}$ & $\begin{array}{r}43 \\
48 \\
51 \\
57 \\
60 \\
66 \\
70 \\
115 \\
198\end{array}$ & $\begin{array}{l}4,3 \\
6,1 \\
6,9 \\
6,6 \\
6,8 \\
6,1 \\
5,7 \\
2,5 \\
1,0\end{array}$ \\
\hline
\end{tabular}

Tab. 2. Berechnung des Mittelwertes und der Streuung für die cancerogene Gesamtdosis „Buttergelb“ in den Versuchsgruppen mit 10, 20 und $30 \mathrm{mg}$.Einzeldosis (nach Mi e s c h e r u. Mitarbb.5).

Dieser Wert für $N$ vom letzten Stichtag ist für die Berechnung der nächsten Prozentzahl $P$ jeweils in Gl. (1) einzusetzen.

Wir benutzten das von $\mathrm{Mi}$ i s cher vorgeschlagene Verfahren für die Berechnung unserer Versuchsergebnisse, obwohl ihm eine gewisse Willkürlichkeit anhaftet. Die erwähnte Störung durch interkurrente Todesfälle zwingt aber dazu, sich für einen Bezugswert zu entscheiden.

Die Ergebnisse der Versuche mit den drei höchsten Dosierungen sind in der Tab. 2 wiedergegeben. Die zur Erzeugung von Leberkrebs bei Ratten notwendige Gesamtdosis „Buttergelb“ $D$ berechnet sich danach für alle drei Dosierungen zu $1020 \mathrm{mg}$.

Der Versuch mit $3 \mathrm{mg}$ ist in die Berechnung der Tab. 2 nicht einbezogen worden, weil die Summen-

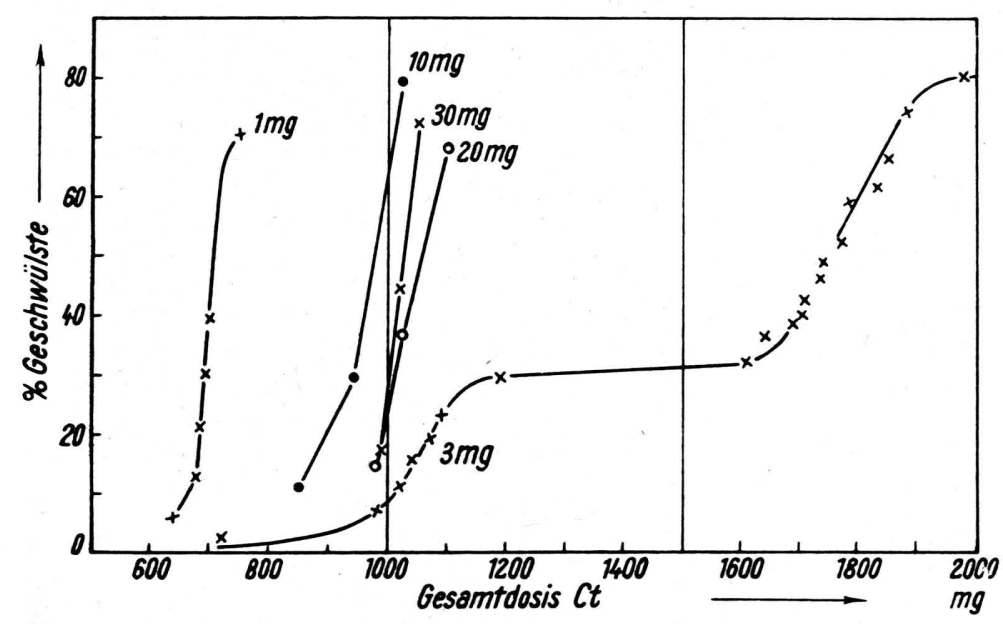

Abb. 1. Summenkurven der beobachteten Geschwulstfälle für die verschiedenen Dosierungen in Abhängigkeit von der für die Geschwulsterzeugung notwendigen Gesamtdosis $(C t)$,Buttergelb“. Berechnung nach Miescher und Mitarbb.5 (vgl. Tab. 3). kurve der Geschwulstfälle hier zwei Stufen hat, die im übrigen charakteristisch sind, nämlich eine mit 7 positiven Fällen zwischen 1000 und 1100 mg Gesamtdosis und eine zweite mit 13 Fällen zwischen 1600 und $1900 \mathrm{mg}$, die zwei verschiedenen Populationen entsprechen. Die hier höhere Zahl der positiven Fälle ist dadurch bedingt, daß allein in diesem Versuch die Latenzzeit kürzer ist als die mittlere Lebenserwartung der Tiere (Tab.1). Die Häufigkeitsverteilung der Geschwulstfälle in den einzelnen Versuchsgruppen geht aus den Summenkurven hervor, die in Abhängigkeit von der Gesamtdosis $C t$ in Abb. 1 dargestellt sind.

Die Berechnung ${ }^{7}$ der Streuung $\sigma= \pm \sqrt{\Sigma x^{2} /(n-1)}$ stößt auf Schwierigkeiten, weil die Zahl der Versuchstiere $n$ gerade in der Zeit, in der die Geschwülste auftreten, auf Grund der toxisch oder natürlich begrenzten Lebenserwartung besonders stark abnimmt. Demgemäß steigt die Streuung der Einzelbeobachtung im Verlauf des Versuchs, und das ,Gewicht der Beobachtungen" 7 n $B$ nimmt ab, wie aus Tab. 2 hervorgeht. Das gleiche gilt für die Berechnung der ,mittleren Abweichung des Mittelwertes “ $\varepsilon= \pm \sigma / V^{\prime} \bar{n}$. Der nach dem ,Gewicht der Beobachtungen" korrigierte Mittelwert für die Streuung ist $\sigma^{\prime}=\boldsymbol{\Sigma}(n B \sigma) / \Sigma(n B)=$ $\pm 63 \mathrm{mg}$ oder $\pm 6 \%$, und für die ,mittlere $\mathrm{Ab}$ weichung des Mittelwertes" $\varepsilon^{\prime}= \pm 18$.

Die gefundene Streuung ist überraschend gering, zumảl bei so langfristigen Versuchen besonders starke Abweichungen verständlich wären. Dem gegenüber steht die immer wieder gemachte Erfahrung, daß bei quantitativen Versuchen mit

7 J. H. Burn, Biologische Auswertungsmethoden. Berlin 1937. 


\begin{tabular}{|c|c|c|c|c|c|c|c|c|}
\hline $\begin{array}{c}D \\
\text { Gesamtdosis }\end{array}$ & $\begin{array}{c}C \\
\text { Einzeldosis }\end{array}$ & $p$ & \multicolumn{2}{|c|}{ positive Fälle } & \multirow{2}{*}{$\mid \begin{array}{c}N \\
\text { negative Fälle } \\
\%\end{array}$} & \multirow{2}{*}{$\begin{array}{c}n \\
\text { Gesamtzahl } \\
\text { Tiere }\end{array}$} & \multirow{2}{*}{$\begin{array}{c}\sigma \\
\text { Streuung } \\
\pm \mathbf{m g}\end{array}$} & \multirow{2}{*}{$\begin{array}{c}n B \\
\text { Gewicht der } \\
\text { Beobachtung }\end{array}$} \\
\hline $\mathrm{mg}$ & & Tiere & $\%$ & $\%$ & & & & \\
\hline $\begin{array}{r}1 \\
365\end{array}$ & $\begin{array}{l}1 \\
1\end{array}$ & $\begin{array}{l}0 \\
0\end{array}$ & $\begin{array}{l}0 \\
0\end{array}$ & $\begin{array}{l}0 \\
0\end{array}$ & $\begin{array}{l}0 \\
0\end{array}$ & $\begin{array}{r}142 \\
53\end{array}$ & & \\
\hline $\begin{array}{l}642 \\
676 \\
685 \\
688 \\
695 \\
747\end{array}$ & $\begin{array}{l}1 \\
1 \\
1 \\
1 \\
1 \\
1\end{array}$ & $\begin{array}{l}1 \\
1 \\
1 \\
1 \\
1 \\
1\end{array}$ & $\begin{array}{r}\mathbf{5 , 6} \\
\mathbf{6 , 7} \\
\mathbf{8 , 8} \\
8,8 \\
8,8 \\
30,7\end{array}$ & $\begin{array}{r}5,6 \\
12,3 \\
21,1 \\
29,9 \\
38,7 \\
69,4\end{array}$ & $\begin{array}{r}100 \\
94,4 \\
87,7 \\
78,9 \\
70,1 \\
61,3 \\
30,6\end{array}$ & $\begin{array}{r}18 \\
14 \\
10 \\
9 \\
8 \\
2\end{array}$ & $\begin{array}{r}21 \\
24 \\
29 \\
30 \\
+32 \\
36\end{array}$ & $\begin{array}{l}4,0 \\
5,4 \\
5,1 \\
5,2 \\
5,0 \\
1,2\end{array}$ \\
\hline
\end{tabular}

Tab. 3. Berechnung des Mittelwertes und der Streuung für die Gesamtdosis „Buttergelb“ im 1-mg-Versuch (nach Miescher u. Mitarbb.5).

krebserregenden Stoffen bei einigermaßen einheitlichen Versuchsbedingungen tatsächlich so merkwürdig geringe Streuungen gefunden werden. Selbst bei Verwendung heterogenen Tiermaterials zeigen die Summenkurven der positiven Fälle gewöhnlich nur zwei oder höchstens drei ganz charakteristische Stufen, wie dies auch $\mathrm{M}$ i e s ch e ${ }^{5}$ gefunden hat. Wir glauben, daß dies Phänomen Beachtung verdient.

Das Ergebnis des 1-mg-Versuch ist in Tab. 3 wiedergegeben. Der Mittelwert der zur Krebserzeugung notwendigen Gesamtdosis beträgt hier nur $705 \pm 28 \mathrm{mg}$, ist also wesentlich kleiner als in allen anderen Versuchsgruppen.

Die „signifikante Differenz" beträgt 16. Damit ist die Differenz zwischen diesem Resultat von $705 \mathrm{mg}$ gegenüber dem von $1020 \mathrm{mg}$ in den anderen Gruppen statistisch gesichert.

Der Befund, daß die cancerogene Gesamtdosis im langfristigen 1-mg-Versuch so erheblich kleiner ist als in den übrigen Versuchen, ist um so auffälliger, als die Gesamtdosis in dem ihm benachbarten 3-mg-Versuch, der durch die größte Zahl der positiven Fälle ausgezeichnet ist, keinesfalls tiefer als $1000 \mathrm{mg}$ liegen kann, sondern nur höher.

\section{Latenzzeit und , $C t-\mathrm{Produkt}$ “}

Die trotz des noch unbefriedigend kleinen Tiermaterials überraschend klaren Ergebnisse zeigen, daß für den so geheimnisvoll erscheinenden Vorgang der Krebsentstehung doch grundsätzlich dieselben Gesetzmäßigkeiten gelten, die auch sonst in der pharmakologischen Forschung als gültig gefunden wurden. Dies gilt vor allem für die
Länge der Latenzzeit. Nach unseren Ergebnissen ist sie eine einfache Funktion der Dosis. Die Einzeldosis bedeutet hier: Menge pro $200 \mathrm{~g}$ Tier, sie hat also die Dimension einer Konzentration. Sehen wir zunächst von der mit der Einzeldosis von $1 \mathrm{mg}$ behandelten Versuchsgruppe ab, so ist in allen Versuchen die Dauer der Latenzzeit, d. h. die bis zum Auftreten der definierten Wirkung $W$ notwendige Behandlungszeit $t$, der Höhe der täglichen Giftdosis $C$ umgekehrt proportional. Es gilt also

$$
C t=\text { const } \quad \text { bzw. } \quad W \sim C t .
$$

Die Dosis-Zeit-Kurve ist in diesem Bereich eine gleichseitige Hyperbel bzw., bei logarithmischer Teilung beider Achsen, eine Gerade mit einem Neigungswinkel von $45^{\circ}$ (Abb. 2). Da die Höhe der Einzeldosis $C$ in jeder Versuchsgruppe konstant gehalten wurde, ergibt das Produkt $\boldsymbol{C} \boldsymbol{t}$.

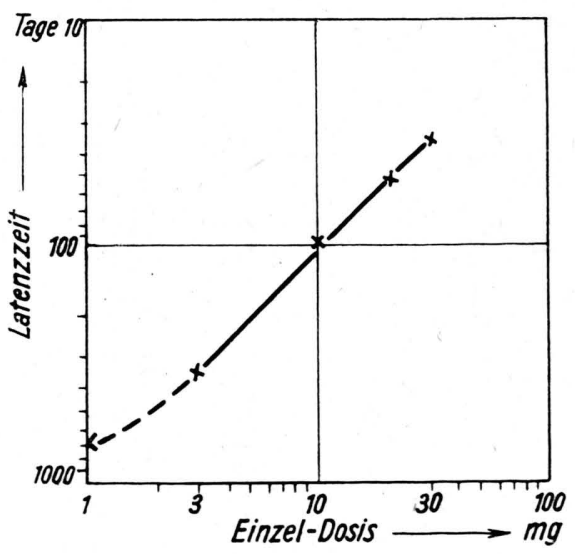

Abb. 2. Abhängigkeit der Latenzzeit bis zum Auftreten der Lebergeschwülste von der Höhe der täglichen Dosis „Buttergelb“. 
die Höhe der Gesamtdosis D. Der Leberkrebs tritt in dem Zeitpunkte auf, in dem die Gesamtdosis $D$ einen kritischen Wert überschreitet, der hier ziemlich genau bei $1000 \mathrm{mg}$ „Buttergelb“ liegt. Dabei ist es gleichgültig, über welche Zeit diese Gesamtdosis verteilt wird.

Versuche mit krebserregenden Stoffen an lebenden Geweben in vivo oder in vitro können danach nur dann Erfolg haben, wenn eine ausreichende Gesamtdosis zur Anwendung kommt. Hohe Konzentrationen führen am schnellsten zum Ziel.

In der Pharmakologie ist eine ganze Reihe von Stoffen bekannt, deren Wirkung durch das Produkt aus der (konstanten) Konzentration $C$ des Giftes und der Behandlungszeit $t$ bestimmt wird, oder, in allgemeiner Formulierung, durch das Integral aus der Giftkonzentration über die Zeit. Sie heißen „Ct$t$-Gifte“.* Die notwendige Voraussetzung für dieses Verhalten ist, daß entweder die Bindung des Giftes am Wirkungsort oder seine Wirkung irreversibel ist, so daß eine Summation der Effekte eintritt. Bei den bisher bekannten „Ct$t$-Giften“ gilt das nur für einen begrenzten Bereich von Zeit und Konzentration, der hinsichtlich der Zeit nur Stunden bis höchstens Tage beträgt. Diese Beschränkung gilt dort, weil über lange Zeit doch eine gewisse Reversibilität oder Reparabilität besteht, so daß bei Anwendung kleiner_Konzentrationen über längere Zeiträume doch höhere Werte für die $C t$-Produkte erhalten werden, als bei kürzerer Versuchsdauer mit höheren Konzentrationen.

Demgegenüber liefern unsere Versuche trotz Ausdehnung über ein Jahr ein konstantes $C t$ Produkt, und bei doppelter Versuchsdauer, die die ganze Lebensdauer der Tiere umfaßt, sogar ein kleineres. Die cancerogene Wirkung des „Buttergelbs" bleibt also auch bei den kleinsten Einzeldosen vom Beginn des Versuchs an auf Lebenszeit irreversibel bestehen und summiert sich mit der Wirkung späterer Gaben ungeschmälert, bis nach Uberschreiten der kritischen Gesamtdosis sich die Geschwülste entwickeln. Dies geschieht bei allen krebserregenden Stoffen nicht allmählich während der Behandlungszeit, sondern sprunghaft. Das anschließende Wachstum der Geschwülste erfolgte auch in diesen Versuchen dann schnell und führte in wenigen Wochen zum Tode des Tieres. Daraus erklärt sich bis zu einem gewissen Grade die geringe Streuung bei Versuchen mit krebserregenden Stoffen. Bei unseren Versuchen entwickelte sich das größte Lebercarcinom in 4. Wochen von Haselnußgröße bis zu einem Gewicht von $102 \mathrm{~g}$.

*.Vgl. H. Druckrey u. K. Küpfmüller, Dosis und Wirkung, Berlin 1948.
Die bei „Buttergelb“-Versuchen zwar stets beobachteten Gallengangswucherungen können nach unseren Erfahrungen nicht als spezifisches Vorstadium des Leberkrebses angesehen werden. Ein sichtbares ,präcanceröses Stadium“ im spezifischen Sinne kennen wir nicht. Das einzige Phänomen, das der Krebsentstehung bei allen krebserregenden Stoffen und Strahlen in gleicher Weise vorauszugehen scheint, besteht in Veränderungen an den Zellkernen, die von $\mathrm{H}$ a m perl genauer untersucht sind und in der „Kernunruhe“, der Störung der Kern-Plasma-Relation und der Kernteilung ihren Ausdruck finden. Sie deuten darauf hin, daß der Angriffspunkt der cancerogenen Wirkung wahrscheinlich an einem Bestandteil des Zellkerns zu suchen ist und daß die cancerogenen Stoffe „Kerngifte“" ${ }^{8}$ sind. Jedoch auch diese Effekte sind insoweit kein spezifisches Merkmal der cancerogenen Wirkung, als sie auch anderen Pharmaka zukommen.

Die Ergebnisse der vorliegenden Versuche zeigen, daß auch kleinste Dosen „Buttergelb“irreversible Wirkungen haben, die, selbst wenn sie zu klein sind, um innerhalb der Lebenserwartung für sich allein Krebs oder auch nur sichtbare Effekte zu erzeugen, doch voll summationsfähig sind und ihrer Art nach auf dem Wege zum Krebs liegen. Die Verwendung des „Buttergelbs“ als Lebensmittelfarbstoff ist deshalb gefährlich und abzulehnen. Darüber hinaus erscheint es nach diesen Befunden notwendig, in der Umwelt des Menschen nach krebserregenden Stoffen zu suchen und vor allem solche auszuschalten, mit denen er täglich in Berührung kommt, und zwar auch dann. wenn die aufgenommene Menge klein ist.

Für die beim „Buttergelb“ gefundene Dosis-Wirkungs-Beziehung gibt es eine interessante und wohl ebenso ernst zu nehmende Parallele in der Mutationen auslösenden Wirkung der Röntgenstrahlen. Die Mutationsrate, also die Anzahl $M_{K}$ von Mutationen, die in einer Population von der $\mathrm{Zahl} M_{0}$ der Individuen auftritt, wird durch die Gesamtstrahlendosis in $r$ bestimmt $M_{K} \sim M_{0} r$ [vgl. Gl. (11)],

ohne Rücksicht auf die Zeit, über die sie verteilt wird, und zwar sogar in einem Dosisbereich, der sich wie 1: 300 verhält ${ }^{9}$. Ob diese Verhältnisse auch für die krebserregende Wirkung der Strahlen zutreffen, ist u. W. noch nicht bekannt. Da beide Strahlenwirkungen irreversible Effekte darstellen, ist auch bei ihnen

$8 \mathrm{~N}$. B r o ck, H. D r u ckrey u. H. Herken, Naunyn-Schmiedebergs Arch. exp. Pathol. Pharmakol. 193, 679 [1939].

${ }^{9}$ A. Cats ch, A. Ranellis, G. Radu u. P. W e lt, Naturwiss. 32, 228 [1944]. 
eine strenge Gültigkeit der Beziehung nach Gl. (3) zu erwarten.

\section{Die Alters-Disposition}

In allen Versuchsgruppen, bei denen sich die Behandlungsdauer bis zu 350 Tagen, also bis zum 450. Lebenstag der Ratten, erstreckte, fanden wir die gleiche Gesamtdosis „Buttergelb“ cancerogen wirksam, nämlich $1000 \mathrm{mg}$. Demgegenüber benötigte die Krebserzeugung im 1-mg-Versuch, der sich über den 800 . Lebenstag, also bis ins hohe dreifachen Zeit folgen, hier also um den 840 . bis 1200. Tag. Das würde einer Gesamtdosis von 250 bis höchstens $400 \mathrm{mg}$ entsprechen, also einem Drittel der sonst zur Krebserzeugung notwendigen Dosis. Diese Latenzzeit fällt aber in die höchste Lebenserwartung unserer Ratten nicht mehr hinein. Es erscheint lohnend, die von uns nur näherungsweise angegebene Kurve der Abb. 3 experimentell genau $\mathrm{zu}$ bestimmen, weil sie wertvolle Einblicke in die Physiologie und Pathologie des Alterns zu gewähren verspricht.

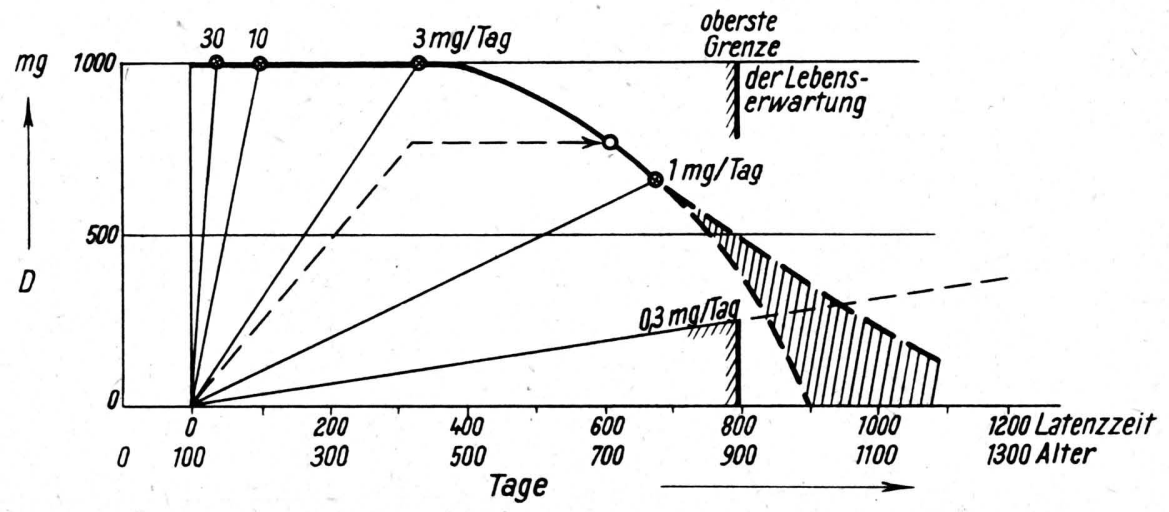

Abb. 3. Abhängigkeit der für die Geschwulsterzeugung notwendigen Gesamtdosis „Buttergelb“ von der Behandlungsdauer bzw. vom Alter der Ratten. Erklärung im Text.

Alter der Ratten, erstreckte, die wesentlich kleinere Gesamtdosis von nur $705 \mathrm{mg}$. Die Differenz ist statistisch gesichert. Die Bereitschaft der Ratten, mit Leberkrebs zu reagieren, ihre „Krebsdisposition", nimmt also mit dem Alter anscheinend ebenso $\mathrm{zu}$, wie dies auch aus der menschlichen Pathologie bekannt ist. Die Abhängigkeit der für die Geschwulsterzeugung notwendigen Gesamtdosis von der Behandlungsdauer bzw. vom Lebensalter ist in Abb. 3 dargestellt. Einen zweiten Anhaltspunkt für die Zunahme der Krebsbereitschaft im Alter und den weiteren Kurvenverlauf in Abb. 3 liefert der Versuch mit $0,3 \mathrm{mg}$ „Buttergelb“. Hier erreichen die Ratten ein Höchstalter von 900 Tagen, haben in 800 Behandlungstagen also $240 \mathrm{mg}$ „Buttergelb“ aufgenommen. Die Kurve der krebserregenden $C t$-Produkte muß also jenseits dieses Punktes vorbeigehen. Die Begrenzung nach der anderen Seite ergibt sich daraus, daß in diesem Versuch nach dem 420. Behandlungstag die als wahrscheinlich unspezifisch bezeichneten Leberveränderungèn auftraten, denen nach unseren Erfahrungen (Tab. 1) die Geschwülste etwa in der doppelten bis höchstens

\section{- 5. Die Krebs-Disposition}

Die Beobachtung, daß die zur Auslösung von Lebergeschwülsten erforderliche Gesamtdosis „Buttergelb“ jenseits eines Lebensalters von 450 Tagen so erheblich abnimmt, deutet darauf hin, daß die Kurve in Abb. 3 schließlich einmal die Abszisse erreicht. In diesem Alter müßte dann schon die Dosis Null krebserzeugend wirken. Das erscheint zunächst absurd, ist aber dennoch erklärlich.

In einer früheren Arbeit ${ }^{10}$ wurde gezeigt, daß das Vorhandensein einer „Disposition“" nicht nur bei der Krebserzeugung eine Rolle spielt, sondern bei jeder pharmakologischen Wirkung. Pharmaka können nur solche biologischen Vorgänge auslösen, die im lebenden Organismus bereits veranlagt, also grundsätzlich auch spontan möglich sind, aber niemals etwas völlig Neuartiges. Die dazu notwendige Dosis ist um so kleiner, je größer die Disposition ist, bis sie bei spontaner Manifestation tatsächlich gleich Null wird. Vgl. z. B. die Wirkung von Follikelhormon bei infantilen und juvenilen Ratten. Demnach ist auch von krebserregenden Stoffen eine Wirkung nur an solchen Tieren und bei diesen nur in den Organen zu erwarten, bei denen auch spontan Geschwülste möglich sind, also eine

10 H. D r u c k r e y, Klin. Wschr. 21, 559 [1942]. 
„Krebsdisposition“ besteht. Die dazu notwendige Dosis wird auch hier um so kleiner sein, je größer die Disposition ist, bis es schließlich zu spontaner Geschwulstbildung kommt. Die Wirkung krebserregender Stoffe besteht demnach darin, daß der Zeitpunkt des Auftretens von spontan möglichen Geschwülsten vorverlegt, der Vorgang beschleunigt wird.

Nach unseren Versuchen wäre ein spontaner Leberkrebs bei Ratten etwa in einem Alter von 1000 bis 1500 Tagen zu erwarten, das allerdings nicht mehr in die Lebenserwartung hineinfällt; er müßte in Erscheinung treten, wenn es gelänge, die Lebenserwartung bei diesen Ratten entsprechend zu erhöhen. Da die mittlere Lebenserwartung des Menschen in den letzten Jahrzehnten beträchtlich gestiegen ist, mag die nachgewiesene größere und zunehmende Häufigkeit bestimmter Arten von Krebs ähnlich zu verstehen sein.

Unsere Beobachtungen zeigen auf jeden Fall, daß die zur Krebserzeugung notwendige Dosis „Buttergelb“ von einem bestimmten mittleren Lebensalter an kleiner wird, die „Krebsdisposition" zunimmt. Damit wird die so verschwommene „Krebsdisposition“ zu einem quantitativ faßbaren Begriff. Die relative Größe des ihr gegenläufigen Wertes, der „Krebsresistenz“, läßt sich zahlenmäßig durch die Dosis angeben, die von einem krebserregenden Stoff notwendig ist, um in diesem Organ Krebs zu erzeugen.

Die Zunahme der Krebsdisposition im Alter kann auch insofern praktische Bedeutung erlangen, als ein unterschwelliger cancerogener Effekt, der ja irreversibel ist, im Alter auf diese Weise einmal überschwellig wird und damit scheinbar „,spontan“ zum Krebs führt, wie es S a s a ki beobachtet hat. Wir haben diesen Sachverhalt in Abb. 3 durch einen gestrichelten Pfeil angedeutet.

Die hier entwickelten Vorstellungen über den Begriff der „Krebsdisposition“ haben eine interessante Parallele in der experimentellen Mutationsforschung. Nach Tim of é f f ${ }^{11}$ werden durch Strahlen in der Regel dieselben Mutationsarten ausgelöst, die auch spontan auftreten; auch ihre relative Zahl wird dabei nicht vermehrt, sondern nur ihr Auftreten verfrüht. Bei neuartig erscheinenden Mutationen wird in diesem Sinne angenommen, daß ihr Auftreten durch die Bestrahlung in den Erlebensbereich hineingezogen wird.

\section{Der Wirkungsmechanis mus}

Das wesentlichste Ergebnis der vorliegenden Versuche ist der Befund, daß zwischen der Höhe

11 N. W. T i m o f e é f f - R e s s o w s k y, Mutationsforschung in der Vererbungslehre. Dresden 1937. Ferner Nova Acta Leopoldina 9, 209 [1940]. der täglichen Dosis „Buttergelb“ und der zur Geschwulsterzeugung notwendigen Behandlungsdauer bis zu einem Jahr die in Gl. (3) ausgedrückte „Ct$t$-Beziehung“ auffallend streng gilt. Die Einfachheit dieser Beziehung legt es nahe, einen einfachen physikalisch-chemischen Prozeß als wesentlich für die Krebserzeugung zu vermuten.

Die cancerogene Wirkung beruht auf einer direkten Einwirkung der krebserregenden Substanz auf die entartende Zelle. Beim „Buttergelb“ erfolgt die Lokalisation des Krebses in der Leber, weil durch die besondere Verteilung (B e n nhold) und Ausscheidung dieses Farbstoffs in diesem Organ die größte Konzentration herrscht und die in Frage kommenden Zellen eine ausreichende Krebsdisposition besitzen.

Die krebsige Entartung der Zellen tritt in dem Zeitpunkte auf, in dem die dem Tier zugeführte Gesamtmenge „Buttergelb“ einen kritischen Wert überschreitet. Die Wirkung muß also darauf beruhen, daß eine bestimmte, im Altersbereich bis zu 450 Tagen konstante Menge eines körpereigenen Stoffes durch das „Buttergelb“ oder durch ein Reaktionsprodukt von ihm verändert wird.

Bezeichnen wir die Anfangskonzentration der spezifischen körpereigenen Substanz (,,spezifische Rezeptoren“), die mit dem „Buttergelb“ reagiert, mit $R$, die Konzentration der bereits veränderten Rezeptoren mit $C_{R}$ und die mittlere Konzentration des „Buttergelbs“, die durch die täglichen Dosen „Buttergelb“ im Tier erzeugt wird, mit $C$, so lautet der allgemeine reaktionskinetische Ansatz für den Fall der bimolekularen Reaktion:

$$
\frac{d C_{R}}{d t}=K\left(R-C_{R}\right) C-\frac{C_{R}}{T_{R}} .
$$

Hierbei ist $K$ die Reaktionskonstante der Kombination und $T_{k}$ die Zeitkonstante der rückläufigen Reaktion. Wir bevorzugen hier den Begriff des "spezifischen Rezeptors" vor dem des reagierenden Moleküls, weil er weiter gefaßt ist und für die makromolekularen Verhältnisse der Zelle besser zutrifft. Der Ansatz in Gl. (4) gilt nicht nur für chemische Reaktionen, sondern auch für die entsprechenden physikalischen Vorgänge, weil er auch statistisch gedeutet werden kann.

Unsere Versuche zeigen nun, daß die cancerogene Wirkung des „Buttergelbs" irreversibel ist. Die Zeitkonstante der rückläufigen Reaktion ist demnach $T_{\boldsymbol{R}}=\infty$ zu setzen, und der Subtrahend 
in Gl. (4) wird Null. Für strenge ..Ct -Gifte" nach Gl. (3) gilt also allgemein:

$$
\frac{d C_{R}}{d t}=K\left(R-C_{R}\right) C .
$$

Unter der hier berechtigt erscheinenden Annahme, daß die Konzentration der veränderten Rezeptoren bis zum Wirkungseintritt klein ist gegenüber der Ausgangskonzentration $R$ der spezifischen Rezeptoren, bleibt $R$ praktisch konstant und die Gl. (5) vereinfacht sich $\mathrm{zu}$ :

$$
\frac{d C_{R}}{d t}=K R C
$$

Da die Dosierung während der Versuchsdauer bei uns konstant gehalten wurde, kann auch die Konzentration $C$ hier als konstant gelten. Dann liefert die Integration der Gl. (6) einfach:

$$
C_{R}=K C R t \text {. }
$$

Das ist die experimentell gefundene $C t$-Beziehung. Die Lösung der Gl. (5) lautet:

$$
C_{R}=1-e^{-K^{\prime} C t} \text {. }
$$

Auch sie entspricht der gefundenen $C t$-Beziehung. Eine eingehendere Darstellung dieser Sachverhalte erfolgte in unserer Monographie „Dosis und Wirkung".

Die Gln. (5), (6) und (7a) geben gleichzeitig den Inhalt der "Treffertheorie" wieder, die ebenfalls auf Wahrscheinlichkeitsüberlegungen beruht ${ }^{12}$. Ihr wesentliches Merkmal liegt demnach auch in der Irreversibilität des Effektes. Es erscheint wichtig, das hervorzuheben.

Die Gl. (7) führt zu einigen Folgerungen. Wenn $C_{R}$ die Menge der durch den krebserregenden Stoff veränderten Rezeptoren pro Gewichtseinheit Tier bezeichnet, deren Überschreiten zum Krebs führt, so wird diese kritische Menge nach Gl. (7) um so früher erreicht, je größer entweder die Menge des Giftes $C$ oder die der spezifischen Rezeptoren $R$ pro Gewichtseinheit Tier ist. Es mu $\beta$ also die Geschwindigkeit der Krebsentstehung von der Menge der spezifischen Rezeptoren pro Tier, d. h. von der relativen Größe des betr. Organs genau so ab-

12 B la u u. A ltenburger, Z. Physik 12, 315 [1923].

${ }_{13}$ Literatur bei C. C. Litt l e, J. Amer. med. Assoc. 1936, 106 . hängen, wie von der Größe der Einzeldosis des krebserregenden Stoffes.

Damit wird eine Erfahrung aufs einfachste verständlich, die sowohl in der experimentellen Krebsforschung wie in der Klinik häufig gemacht wurde, und die bisher einer befriedigenden Deutung nicht zugänglich war. Es erkranken z. B. bei erblich für Brustkrebs anfälligen Mäusestämmen (M. S l y e) nur die weiblichen Tiere. Wird aber bei männlichen Mäusen der gleichen Stämme die Brustdrüse durch oestrogene Stoffe beliebiger chemischer Konstitution zur Entwicklung gebracht, so erkranken auch sie (Lacas sagne). Umgekehrt bleiben die anfälligen Weibchen verschont, wenn sie rechtzeitig kastriert werden; die Brustdrüse atrophiert ( $\mathrm{L}$ o e b und $\mathrm{M} \mathrm{u}$ r r a y) ${ }^{13}$. Das Entscheidende ist demnach wohl die relative Größe der Brustdrüse, die der Wirkung des von B it t $n$ e $\mathrm{r}^{14}$ bei diesen Tieren gefundenen endogenen krebserregenden Stoffs ausgesetzt ist. Die immer wieder geäußerte Meinung, das Follikelhormon habe cancerogene Wirkung, ist mehrfach widerlegt worden ${ }^{15}$.

Solche Zusammenhänge scheinen auch in der menschlichen Pathologie eine Rolle zu spielen. Die Erfahrung hat z. B. gezeigt, daß die Häufigkeit des Brustkrebses bis zu einem gewissen Alter zunimmt, dann aber mit der nun folgenden Involution der Drüse wieder seltener wird. Ähnliches gilt für den Genitalkrebs und die Geschwülste mesenchymalen Ursprungs, deren Muttergewebe im Alter ebenfalls zurückgebildet wird. Beim Mann wurde nach hohen Dosen von Follikelhormon nicht nur eine Entwicklung der Brustdrüse, sondern gelegentlich auch Brustkrebs beobachtet. Auch die in neuerer Zeit erhobene Feststellung, daß nach Behandlung mit Leberpräparaten auffällig oft Magenkrebs auftritt, wird durch die so bewirkte Volumzunahme der Magenschleimhaut zwangloser erklärt als durch die im übrigen unbewiesene Annahme einer cancerogenen Wirkung der Leberpräparate. Wir glauben, daß diese Zusammenhänge, die sich uns weiter unten nochmals aufdrängen, für die Klinik des menschlichen Krebses Beachtung verdienen.

Die Zunahme der Krebsdisposition im Alter, die wir bei unseren Versuchen fanden, kann nach Gl. (7) folgende Ursachen haben:

a) Die zur Krebserzeugung notwendige kritische Menge der durch den krebserregenden Stoff veränderten spezifischen Rezeptoren $C_{k}$ pro Gewichts. einheit Tier wird im Alter kleiner.

b) Die relative Menge der körpereigenen Rezeptoren $R$, an denen die cancerogene Wirkung angreift, nimmt im Alter zu.

c) Die Konzentration des krebserregenden Stoffes $C$ am Wirkungsort nimmt im Alter zu, z. B.

14 J. J. B i t t n e r, Cancer Res. 1, 113 u. 793 [1941]; 3, 441 [1943]; 4, 159 [1944].

15 H. Fried ri ch-Freks a, Ber. Gynäkol. Geburtshilfe 40, 225 [1940]; H. D r u c k r e y, Z. Krebsforschg. 50, 27 [1939]. 
durch Hinzukommen einer endogenen cancerogenen Substanz oder durch eine veränderte Giftverteilung.

d) Die Reaktionsfähigkeit der spezifischen körpereigenen Rezeptoren, d. h. die Reaktionskonstante $K$, wird größer.

Alle vier aufgeführten Möglichkeiten' sind in gleichem Maße vorstellbar und dürften ganz allgemein das präzisieren, was sonst verschwommen genug als „Disposition“ bezeichnet wird.

Hinsichtlich der Natur der spezifischen Rezeptoren sind zwei Möglichkeiten denkbar: entweder ist es ein fester Zellbestandteil, dessen ausreichende Veränderung durch einen krebserregenden Stoff selbst zum Krebs führt, oder der Stoff wirkt einer spontanen Neigung zur krebsigen Entartung entgegen. Sein Vorrat im Organismus müßte, da in unseren Versuchen stets eine bestimmte Gesamtdosis „Buttergelb“ zum Krebs führte, begrenzt sein und durch die Wirkung des cancerogenen Agens verbraucht werden. Beide Möglichkeiten sind denkbar.

\section{Gegenstoff-Theorie}

Die letztgenannte Vorstellung, die hier kurz als „Gegenstoff-Theorie" bezeichnet werden soll, ist nach unseren Ergebnissen nur unter bestimmten Voraussetzungen diskutabel. Die Krebsentstehung wäre nach dieser Theorie in zwei Vorgänge zerlegt zu denken, nämlich erstens in die Beseitigung des Gegenstoffes durch ein cancerogenes Agens, und zweitens in die eigentliche Krebsentstehung, die durch den Ablauf des ersten Vorgangs möglich geworden ist. Der zweite Vorgang müßte dann rein endogen bedingt, seine Geschwindigkeit also unabhängig sein von der Dosierung des cancerogenen Stoffes. Er müßte aber die für den ersten Vorgang gefundene Beziehung $C t=$ const um so mehr stören, je kürzer die Latenzzeit ist. Wir fanden aber gerade bei den kurzen Latenzzeiten ein auffallend konstantes $C t$-Produkt. Demnach müßte der zweite Vorgang der eigentlichen Krebsentstehung praktisch zeitlos erfolgen, oder es müßten ständig Krebszellen im Organismus „spontan“ entstehen. Das scheint allerdings möglich zu sein.

Die Notwendigkeit einer weiteren Voraussetzung folgt daraus, daß die Krebsdisposition der verschiedenen Gewebe an ein und demselben Tier bekanntlich verschieden ist. Demnach müßte es ver- schiedene organspezifische „Gegenstoffe" geben, deren Vorrat im Organismus, der unterschiedlichen Disposition entsprechend, verschieden ist. Dafür gibt es Argumente. So haben japanische Autoren gezeigt, daß das Auftreten von Leberkrebs durch Leberextrakte verhindert werden kann. Wir haben in Versuchen mit Leberfütterung Ahnliches gesehen, allein unser Beobachtungsmaterial ist zu klein für irgendwelche Aussagen. Wegen der großen therapeutischen Bedeutung dieses Problems wäre seine experimentelle Klarstellung erwünscht.

Aus einem weiteren Grunde kann die ,Gegenstoff-Theorie" nicht übergangen werden. Wird an Stelle der Annahme eines in begrenzter Menge vorhandenen organspezifischen „Gegenstoffes“ ein begrenztes Regenerationsvermögen gesetzt, dessen Erschöpfung durch chronische Schädigung zum Krebs führt, so ist damit etwa die Vi r c h o w sche Krebstheorie ausgedrückt. Wir werden später noch auf die „Gegenstoff-Theorie“ zurückkommen.

\section{Rezeptoren-The orie}

Im folgenden wird die andere der beiden Möglichkeiten vertreten, daß nämlich in jeder Zelle „spezifische Rezeptoren“ vorhanden sind, deren Veränderung durch einen cancerogenen Stoff direkt zur krebsigen Entartung der Zelle führt.

Hinsichtlich der Natur dieser „Rezeptoren“ ergeben unsere Beobachtungen zwangsläufig einige Folgerungen. In der jahrelangen Versuchsdauer finden häufig Zellteilungen statt, so daß später gegebene Einzeldosen nicht mehr dieselben Zellen treffen wie die ersten, sondern ihre Tochterzellen. Wenn nun mit der Zellteilung jedesmal eine Division des Effektes eintreten würde, dann müßte die zur Krebserzeugung notwendige Gesamtdosis um so mehr zunehmen, je häufiger sich die Zellen inzwischen geteilt haben, je größer also die Latenzzeit im Versuch war. Unsere Versuche ergaben aber gerade bei der längsten Versuchsdauer die kleinste Gesamtdosis. Die Einzeleffekte sind daher auch in den Tochterzellen noch voll summationsfähig vorhanden, sie haben sich bei den Zellteilungen nicht ,verdünnt“, sondern an der Reduplikation teilgenommen. Die spezifischen Rezeptoren der Zelle, an denen die cancerogenen Agenzien angreifen, müssen also zur Selbstreproduktion befähigte Substanzen sein, die die Wirkung von den ersten Stadien an ungeschmälert auf die Tochterzellen 
übertragen, also vererben, wie es die voll cancerisierte Zelle ja sicher tut ${ }^{16}$. Diese Annahme ist nicht willkürlich, sondern folgt notwendig aus unseren experimentellen Ergebnissen. Aus diesem Grunde bezeichnen wir die krebserregenden Stoffe nach der Art ihrer Wirkung als „Erbänderungsstoffe“. Die Stärke ihrer Wirkung muß, in allgemeiner Formulierung, einerseits gro $\beta$ genug sein, um an Zellen erbliche Veränderungen in einem für ein autonomes Verhalten genügenden Umfang zu erzeugen, andererseits muß sie aber nicht ausreichend sein für die Schaffung letaler Effekte.

Es erscheint notwendig, diese Folgerungen gegenüber der „Mutationstheorie“" der Krebsentstehung klar zu umreißen. Eine Mutation ist eine erbliche Veränderung an Genen oder Chromosomen. Eine Genmutation stellt ein monomolekulares Ereignis dar, das durch einen einzigen ,,Treffer" auslösbar ist. Eine derartig feine Veränderung an einer somatischen Zelle mit ihrem diploiden Chromosomensatz würde für ein malignes Wachstum nicht im entferntesten ausreichen. Für die cancerogene Erbänderung der Zelle wissen wir demgegenüber nicht, ob sie an chromosomalen Elementen oder an anderen, zur Selbstproduktion befähigten Substanzen des Zellkerns oder vielleicht auch des Cytoplasmas angreift. Aber der Umfang dieser Veränderung muß weit größer sein als der bei einer Genmutation, um eine genügende Autonomie der betroffenen Zellen im Gewebe herbeizuführen; er kann auch viel größer sein, weil die Krebszelle ja nur auf gegebenem Nährboden zu vegetieren braucht. Das eine zeigen jedenfalls alle histologischen Bilder von Krebsgeschwülsten zweifelsfrei, daß derartige Zellteilungen nicht mehr zu einem geordneten Organ, geschweige denn zu einem lebensfähigen komplizierten Organismus führen können, wie dies mutierte Keimzellen können, deren Zellteilungen und Organbildungen zum Unterschied von denen der Krebszellen völlig geordnet verlaufen. Zwischen Mutation und krebsiger Entartung besteht zumindest ein gewaltiger quantitativer Unterschied, wie etwa zwischen einem physiologischen Vorgang und seiner pathologischen Entgleisung.

Eine krebsige Entartung wird um so stärker sein, je mehr die „spezifischen Rezeptoren“ durch das cancerogene Agens verändert wurden, von gutartigen Geschwülsten angefangen bis zu höchst bösartigem Krebs ${ }^{16}$. Das entspricht der experimen-

${ }^{16}$ H. D r u c k r e y, Klin. Wschr. 15, 401 [1936]. tellen Erfahrung auch in diesen Versuchen. Dabei haben wir den Eindruck, daß bestimmte Stufen der Entartung bevorzugt sind.

Wir nehmen im Sinne der oben gegebenen allgemeinen Formulierung an, daß eine Krebszelle entsteht, wenn die Anzahl $n_{K}$ der spezifischen Rezeptoren einer Zelle durch den krebserregenden Stoff verändert ist, gleichzeitig aber $n_{L}$ bestimmte andere Rezeptoren, deren Veränderung letal sein würde, nicht betroffen sind. Die Wahrscheinlichkeit $W$ der Besetzung eines Rezeptors wächst während eines Zeitelementes $d t$ um einen Betrag, der um so größer ist, je höher die Konzentration des krebserregenden Stoffes ist und je mehr unveränderte Rezeptoren noch vorhanden sind. Dies führt zu Ansätzen von der Form der Gln. (5), (6) und (7a), da die Wirkung irreversibel ist, wobei $W$ an Stelle von $C_{R}$ zu setzen ist.

Die Wahrscheinlichkeit für das Entstehen einer Krebszelle ist dann

$$
W_{K}=W^{n_{K}}(1-W)^{n_{L}} .
$$

Diese Wahrscheinlichkeit hat ein Maximum

$$
W_{K \max }=\frac{n_{K}^{n_{K}} n_{L}^{n_{L}}}{\left(n_{K}+n_{L}\right)^{n_{K}+n_{L}}}
$$

bei

$$
t_{\max }=\frac{1}{K C} \ln \left(1+\frac{n_{K}}{n_{L}}\right) .
$$

Die Bildung der Krebszellen ist also auf einen bestimmten Zeitabschnitt beschränkt. Vorher reicht die Wahrscheinlichkeit der Besetzung der $n_{K}$-Rezeptoren nicht aus, während es nachher nicht mehr wahrscheinlich genug ist, daß die $n_{L}$-Rezeptoren unverändert bleiben. Das kann z. B. ein Grund dafür sein, daß bei manchen Geschwulstarten die Häufigkeit des Vorkommens mit dem Alter nicht kontinuierlich zunimmt.

\section{Z a hlenmäßige Näh erung}

Sind in einem Organ, wie z. B. der Leber, $N_{0}$ Zellen vorhanden, so beträgt die Zahl der erzeugten Krebszellen im Zeitpunkt $t$

$$
N_{K}=N_{0} W_{K} \text {. }
$$

Sie wächst also um so schneller, je größer die Anzahl der Zellen $N_{0}$ im betroffenen Organ ist. Damit kommen wir hier zu demselben Ergebnis, zu dem schon die reaktionskinetischen Überlegungen 
geführt hatten, daß nämlich die relative Größe des Organs maßgeblich für die Geschwindigkeit der Krebsentstehung sein muß.

Nun sind zwei aufeinanderfolgende Vorgänge scharf zu unterscheiden. Der erste besteht in der Erzeugung des Geschwulstkeimes aus normalen Körperzellen durch ein cancerogenes Agens. An ihn schließt sich dann der zweite, das eigentliche Wachstum der Geschwulst, an, das nun autonom ist und des Weiterwirkens der krebserzeugenden Ursache nicht mehr bedarf. Die Malignität ist eine Eigenschaft der Zelle selbst geworden, und zwar eine irreversible erbliche ${ }^{16}$. Vgl. hierzu die Virusgeschwülste, bei denen dieser Nachweis fehlt.

Für das Wachstum der Geschwulst aus den erzeugten Krebszellen durch Zellteilung bestehen zwei Möglichkeiten. Entweder ist hierzu eine einzige Krebszelle als Geschwulstkeim ausreichend, oder es ist eine bestimmte Mindestzahl von Krebszellen $N_{K \text { min }}>1$ erforderlich. Auf Grund unserer Versuchsergebnisse läßt sich hier eine Entscheidung treffen.

Der Zeitpunkt, in dem die erste Krebszelle nach dem hier vorausgesetzten Mechanismus entsteht, ist naturgemäß sehr stark vom Zufall abhängig. Bei $N_{K \min }=1$ müßte demnach die Streuung sehr groß sein. Die Verhältnisse entsprechen etwa der Frage, wie oft man würfeln muß, um eine $6 \mathrm{zu}$ werfen. Dies kann schon beim ersten Wurf geschehen, aber ebensogut können 10 oder gar 20 Würfe dazu nötig sein. Um dagegen 1000-mal eine $6 \mathrm{zu}$ werfen, werden schon ziemlich genau 6000 Würfe nötig sein. Die durch den Zufall bedingte „mittlere Streuung“ ist in unserem Falle

$$
\sigma=\sqrt{N_{0} W_{K}}=\sqrt{N_{K \min }} .
$$

Die folgende Tabelle gibt diese Abweichungen für einige Werte von $N_{K \min }$ in Prozenten von $N_{K \min }$ an:

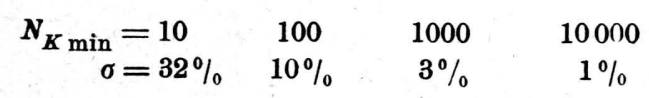

Die berechnete Streuung o besagt, daß in $37 \%$ aller Fälle die Zahl der erzeugten Krebszellen um mehr als 5\% von der nach Gl. (11) zu erwartenden Anzahl abweichen kann.

In unseren Versuchen betrug die Gesamtstreuung, in der also noch andere mit einer Streuung belastete Vorgänge enthalten sind, etwa 7\%. Da die Streuung des hier betrachteten Einzelvorganges kleiner sein muß, folgt, daß die zum Geschwulstwachstum erforderliche Mindestzahl von Krebszellen wahrscheinlich größer als 100 oder sogar 1000 sein wird. Dies entspricht auch der Erfahrung. Z. B. sind für die erfolgreiche Verimpfung des J e n s e n - Sarkoms mindestens 100000 Krebszellen notwendig ${ }^{16}$, allerdings bei hohem Gehalt an nicht mehr teilungsfähigen Zellen und an einem völlig gesunden Tier.

Die gezogene Folgerung über die Größe von $N_{K \text { min }}$ führt zu neuen Gesichtspunkten für das Metastasen-Problem und zu einer weiteren Erklärungsmöglichkeit für die Zunahme der Krebsdisposition im Alter, nämlich der, daß die kritische Mindestzahl hier kleiner wird. Das deckt sich praktisch mit der im Punkt a) auf S. 262 behandelten Möglichkeit.

Die naheliegende Frage, auf welche Weise das Überschreiten von $N_{\boldsymbol{K} \text { min }}$ die Voraussetzung zum Geschwulstwachstum schafft, soll hier nicht näher untersucht werden. Jedoch folgt aus dem berechneten Wert für $N_{\boldsymbol{K} \text { min }}$ eine Einschränkung des möglichen Bereichs der beiden Rezeptoren-Arten $n_{K}$ und $n_{L}$. Es muß nämlich der Maximalwert der Wahrscheinlichkeit für die Entstehung von Krebszellen $W_{K \text { max }}$ mindestens so groß sein, daß $N_{K \text { min }}$ erreicht wird. Danach läßt sich mit Gl. (9) berechnen, in welchem Bereich die Zahlenwerte für $n_{K}$ und $n_{L}$ liegen können. Wird größenordnungsmäßig $N_{K \text { min }}=10^{3}$ angenommen und die Zahl der Leberzellen bei der Ratte mit $N_{0}=10^{9}$, so folgt nach Gl. (11) $W_{K \max }=10^{-6}$. Das Ergebnis dieser Berechnung ist in Abb. 4 dargestellt. Wird als Mindestwert für die Anzahl der beiden RezeptorenArten die Zahl 2 angenommen, dann liegen die

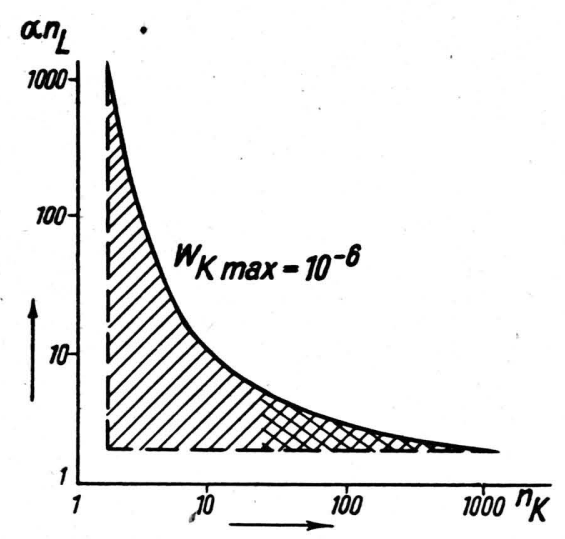

Abb. 4. Mögliche Wertepaare für die beiden Rezeptoren-Arten $n_{K}$ und $n_{L}$. Erklärung im Text. 
möglichen Werte von $n_{K}$ und $n_{L}$ auf dem gezeichneten Kurvenstück bzw. bei $W_{K \text { max }}>10^{-6}$ in dem schraffierten Bereich. Es sind also nur ziemlich eng begrenzte Wertepaare möglich. Dies gilt jedoch nur, wenn die Reaktionskonstanten für beide Rezeptoren-Arten einander gleich sind, wenn ihr Quotient $\alpha=1$ ist. Dagegen gilt auch bei beliebigen Werten von $\alpha$ gemäß Abb. 4 immer, daß bei großen Werten von $n_{K}$ die Werte von $\alpha n_{L}$ klein sein müssen und umgekehrt.

Die Größe der Latenzzeit wird in erster Linie durch die Anzahl der $n_{K}$-Rezeptoren bestimmt. Wenn ihre Anzahl $n_{K}=1$ ist, genügt eine Wahrscheinlichkeit der Rezeptorenveränderung von $W=10^{-6}$, damit $W_{K}=10^{-6}$ wird und von den $N_{0}=10^{9}$ Leberzellen nach Gl. (11) in $N_{K \text { min }}=10^{3}$ Zellen der eine spezifische Rezeptor verändert wird. Diese bei $n_{K}=1$ erforderliche Wahrscheinlichkeit $W$ wird in einem Zeitpunkt $t$ erreicht, für den nach Gl. (3) bzw. (7) $K C t=10^{-6}$ bzw. $K R C t=10^{-6}$ ist.

Ist dagegen $n_{K}=2$, so muß die Wahrscheinlichkeit der Rezeptorenveränderung $W=K C t$ den höheren Wert $10^{-3}$ haben, damit wieder die Wahrscheinlichkeit der Entstehung von Krebszellen $W_{K}=10^{-6}$ wird. Es genügt dann also nicht, daß insgesamt 2000 beliebige Rezeptoren verändert werden, damit $1000 \mathrm{Krebszellen}$ entstehen, sondern es müssen von den angenommenen $2 \cdot 10^{9}$ in den Leberzellen vorhandenen spezifischen Rezeptoren dieser Art 2000000 durch den cancerogenen Stoff verändert sein; dann erst sind in 1000 Leberzellen die beiden speziellen Rezeptoren betroffen. Bei $n_{K}=2$ ist die Latenzzeit also 1000-mal länger als bei $n_{K}=1$. Bei weiterer Zunahme von $n_{K}$ wächst die Latenzzeit weiter an, wenn auch weniger stark. Die bei der Krebserzeugung gesetzmäßig langen Latenzzeiten erklären sich also nur, wenn $n_{K}$ in dem doppelt schraffierten Bereich von Abb. 4 liegt, d. h. groß ist gegenüber $\alpha n_{L}$. Bei Genmutationen liegt dagegen ein Eintreffer-Ereignis vor, demgemäß sind die dort beobachteten Latenzzeiten sehr viel kürzer als bei der Krebserzeugung. Ein systematischer Vergleich der bei beiden Vorgängen beobachteten Latenzzeiten könnte hier weitere Aufschlüsse geben.

Für den Fall der Entstehung letaler Effekte durch Veränderung der $n_{L}$-Rezeptoren gelten grundsätzlich die oben angeführten Überlegungen und Rechnungsansätze sinngemäß auch. Da krebserzeugende Stoffe nun erfahrungsgemäß viel häufiger und auch frühzeitiger letale Effekte an den Zellen setzen, als sie krebsige Entartung herbeiführen, scheint für den Zelltod bereits die Veränderung von sehr wenigen $n_{L}$-Rezeptoren zu genügen bzw. deren Reaktionskonstante größer zu sein, was dasselbe bedeutet. Nach der in Abb. 4 wiedergegebenen Beziehung zwischen der Anzahl der $n_{K^{-}}$und $n_{L}$-Rezeptoren ist also auch aus diesem Grunde mit einer größeren Zahl der $n_{K}$-Rezeptoren, die für eine krebsige Entartung verändert sein müssen, zu rechnen, jedenfalls mit einer größeren Zahl, als bei Keimzellen Mutationen in Betracht kommen.

Wird angenommen, daß die Besetzung der $n_{K^{-}}$ Rezeptoren durch einen organspezifischen körpereigenen Stoff, dessen Vorrat begrenzt ist, verhindert wird, so liegt im Prinzip ein spezieller Fall der in der „Gegenstoff-Theorie“ behandelten Vorstellungen vor. 